\title{
Situation actuelle et perspectives des systèmes d'autorégulation des entreprises en Amérique latine
}

Diego Marcelo Ledesma Iturbide

\section{(2) OpenEdition Journals}

Édition électronique

URL : https://journals.openedition.org/rdctss/1181

DOI : $10.4000 /$ rdctss. 1181

ISSN : 2262-9815

Éditeur

Centre de droit comparé du travail et de la sécurité sociale

Édition imprimée

Date de publication : 1 avril 2020

Pagination : 56-65

ISSN : 2117-4350

\section{Référence électronique}

Diego Marcelo Ledesma Iturbide, «Situation actuelle et perspectives des systèmes d'autorégulation des entreprises en Amérique latine », Revue de droit comparé du travail et de la sécurité sociale [En ligne], 1 | 2020, mis en ligne le 01 novembre 2021, consulté le 11 novembre 2021. URL : http:// journals.openedition.org/rdctss/1181; DOI : https://doi.org/10.4000/rdctss.1181

\section{(c)}

Revue de droit comparé du travail et de la sécurité sociale est mise à disposition selon les termes de la Licence Creative Commons Attribution - Pas d'Utilisation Commerciale - Pas de Modification 4.0 International. 


\title{
SitUATION ACTUELLE ET PERSPECTIVES DES SYSTÈMES D'AUTORÉGULATION DES ENTREPRISES EN AMÉRIQUE LATINE
}

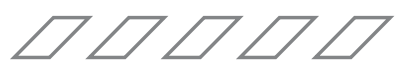

\begin{abstract}
RÉSUMÉ
L'autorégulation des entreprises repose sur des instruments conçus et mis en œuvre de façon volontaire; elle se veut la plus exhaustive possible afin de prémunir l'organisation contre tous les risques dits de non-conformité juridique. Ces mécanismes d'autorégulation suscitent actuellement un intérêt croissant à l'échelle mondiale. Des normes sont fixées et doivent être respectées afin d'obtenir de meilleurs résultats en termes d'efficacité et d'efficience. Par ailleurs, au regard du droit international des droits de l'homme, l'entreprise est directement responsable du non-respect des dispositions de celui-ci, elle est donc tenue au devoir de diligence raisonnable. Cet article vise à faire le lien entre ces questions, et à décrire la situation juridique actuelle en Amérique latine, caractérisée par l'application effective des principes de l'Etat de droit constitutionnel et conventionnel. L'objectif consiste également à décrire la façon dont cette situation incite les autorités judiciaires de chaque État à demander à l'entreprise de rendre compte des mesures de diligence raisonnable mises en œuvre ou de l'existence de systèmes adéquats de protection et de prévention des risques, en cas d'examen de sa responsabilité pour d'éventuelles violations des droits de l'homme ou dommages à l'environnement.
\end{abstract}

MOTS CLÉS: Conformité, diligence raisonnable, droit international des droits de l'homme, État de droit constitutionnel et conventionnel.

\begin{abstract}
Business self-regulation systems are voluntary design and implementation instruments and are intended to be comprehensive, in the sense that they are ideal for warning the organization against all the so-called legal non-compliance risks. These mechanisms are currently of growing global interest. Standards are set and should be met in order to achieve better results in terms of effectiveness and efficiency. On the other hand, transnational human rights law holds the company directly responsible for not complying with their regulations and, in turn, it requires it to practice due diligence within the organization itself. This contribution seeks to link these issues and to describe the current legal situation in Latin America, characterized by the effective application of the principles of the constitutional and conventional rule of law, and to state how this situation encourages the jurisdictional authority in each State to request the organization to account for its due diligence practices or the implementation of adequate systems of protection and risk prevention in processes where the responsibility of the company for possible human rights violations or environmental damage is being discussed.
\end{abstract}

KEY WORDS: Compliance, Due diligence, Transnational Human Rights Law, Constitutional and Conventional State of Law. 


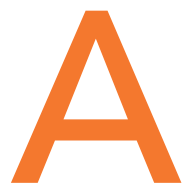

près avoir analysé les raisons qui ont conduit à l'essor international des systèmes d'autorégulation des entreprises, puis examiné la conformité de ces systèmes avec le droit international des droits de l'homme (I), la présente contribution s'attardera plus spécifiquement sur les attentes des États en matière de droit international des droits de l'homme au regard notamment de son impact sur l'activité juridictionnelle des États (II).

\section{I - L'AUTORÉGULATION AU SERVICE DU DROIT INTERNATIONAL DES DROITS DE L'HOMME}

\section{A - L'ESSOR INTERNATIONAL DES ENTREPRISES}

Les systèmes d'autorégulation des entreprises sont des instruments dont la portée, l'efficacité et l'efficience dépendent de l'intérêt personnel de celui qui les conçoit et les met en œuvre, et qui généralement aussi en assure seul le contrôle et la supervision. Cette dimension éminemment volontaire et personnelle de l'outil, cette absence intrinsèque d'hétéronomie, conduit naturellement à sa disqualification comme dispositif adéquat ou idéal pour garantir le plein respect des obligations légales auxquelles l'organisation elle-même décide de se plier. En outre, l'autorégulation est généralement considérée comme un choix commercial par lequel l'entreprise vise à satisfaire uniquement ses propres intérêts.

Malgré cette faiblesse associée ou inhérente au mécanisme, son développement suscite un intérêt mondial croissant qui mérite qu'on y prête attention. II semblerait que l'explication soit avant tout d'ordre économique, liée à la volonté de part et d'autre, d'adopter et de promouvoir des politiques efficaces visant à éradiquer la corruption dans les transactions commerciales internationales, et à garantir le respect des principes du libre-échange.

Ceci conduit à la mise en œuvre d'actions et de réglementations sur l'éthique publique et la lutte contre la corruption, dans les domaines de prévention et de lutte contre les actes criminels en matière économique et financière (corruption, pots-de-vin, contrebande, blanchiment d'argent, etc.). Ces réglementations sont prônées par des entités telles que l'Organisation de Coopération et de Développement Economiques (OCDE) et le Groupe d'action financière (GAFI), ainsi que I'Organisation des Nations Unies (ONU), I'Union Européenne et l'Organisation des États américains (OEA). Cette question occupe également une place de choix dans le dialogue intergouvernemental, comme le G20, et rencontre un large écho au sein des pays d'Amérique latine.

Comme indiqué ci-avant, toutes ces politiques expliquent l'intérêt croissant pour les systèmes d'autorégulation des entreprises dans la mesure où ils ne s'adressent pas seulement aux États et à leurs fonctionnaires, mais visent également la personne morale 
privée, l'entreprise, avec une législation qui engage sa responsabilité civile, administrative, voire pénale dans les affaires de criminalité économique?

Les entreprises privées sont tenues non seulement de coopérer dans le cadre des enquêtes, mais aussi de lutter contre les pratiques frauduleuses au sein de leur propre organisation, sous peine de voir leur responsabilité mise en cause. C'est précisément ce dernier point qui explique l'intérêt suscité par l'autorégulation; une doctrine qui se nourrit et s'inspire de la notion de Responsabilité Sociale des Entreprises (RSE).

Plusieurs États d'Amérique latine ont des réglementations qui engagent la responsabilité administrative et même pénale des personnes morales privées pour des actes de corruption ou des crimes économiques (Brésil, Colombie, Bolivie, Chili, Équateur, Pérou, Mexique et Argentine). La plupart de ces dispositions prévoient que l'existence même d'un système adéquat de conformité réglementaire ou de prévention de la criminalité constitue une circonstance atténuante, voire un motif d'exonération de responsabilité au moment de fixer une peine ${ }^{2}$. Force est de constater ici l'importance de mettre en place de tels programmes ou systèmes dans la région. Mais cet aspect n'est bien sûr pas leur seule raison d'être puisque, dans certains cas, ils répondent à une exigence légale pour pouvoir participer à des appels d'offres ${ }^{3}$, ou nouer des partenariats avec des tiers (fournisseurs, investisseurs, etc. $)^{4}$.

L'importance et la place attribuées à ces programmes de conformité volontaires ont suscité l'intérêt de la communauté internationale quant au développement de ce type de mécanisme, et à la définition des exigences ou des normes auxquelles ils devraient répondre. L'objectif étant qu'ils soient appréhendés, non pas comme une simple formalité, mais de façon sérieuse et au regard de leur efficacité. En ce sens, il existe un certain nombre de standards reconnus au niveau international, parmi lesquels l'Évaluation des programmes de conformité publiée par la Division pénale du Département de la Justice américain, le Guide des bonnes pratiques pour les contrôles internes, la déontologie et la conformité, le Manuel de déontologie et de conformité contre la corruption de l'OCDE, et le Programme de déontologie et de conformité contre la corruption pour les entreprises de l'ONUDC. Les normes internationales ISO 19600 sur les systèmes de management de la compliance, et ISO 37001 sur les systèmes de management anti-corruption ont également acquis une certaine notoriété. doit :

D’une manière générale, il est possible de dire qu'un bon système d'autorégulation

a) être adapté à l'entreprise, c'est-à-dire tenir compte de sa taille, de sa capacité économique et des risques spécifiques et particuliers auxquels elle est exposée ;

1 Cette prévention est une exigence de la Convention de l'OCDE sur la lutte contre la corruption d'agents publics étrangers dans les transactions commerciales internationales (art. 2), de la Convention des Nations Unies contre la corruption (art. 26) et de la Convention interaméricaine contre la corruption (art. VIII).

2 En Argentine, voir art. 9.b de la loi n² 27.401; au Chili, voir art. 3 et 4 de la loi 20.393 ; au Brésil, voir art. 18.5 du décret $n^{\circ} 8420 / 2015$.

3 En Argentine, voir l'article 23 de la loi n 27401.

4 Au-delà de ces dispositifs d'ordre juridique ou relatifs aux liens inter-entreprises, il faut souligner, à juste titre, les bénéfices commerciaux que l'entreprise peut tirer d'une meilleure réputation, d'une production plus durable, ou encore d'une meilleure gouvernance interne, laquelle est en soi une avancée précieuse. 
b) faciliter l'identification de comportements incongrus au sein même de l'organisation et, en ce sens, rompre le " pacte du silence » et favoriser la mise en place d'un système de signalement interne, avec des garanties suffisantes pour la sécurité du plaignant et la protection adéquate de la confidentialité des données collectées;

c) bénéficier d'un soutien au plus haut niveau de la direction et de personnes désignées responsables de la conformité en interne ;

d) organiser une communication périodique et l'élaboration de programmes de formation spécifiques ;

e) compter sur des procédures et des mesures en matière de diligence raisonnable applicables également aux tiers avec lesquels des relations sont établies ;

f) prévoir des instruments de contrôle de leur efficacité et de leur fonctionnement ;

g) être mis à jour régulièrement ;

h) pouvoir être justifié comme étant raisonnable, sur demande des autorités ${ }^{5}$.

\section{B - LES SYSTÈMES D'AUTORÉGULATION, UNE RÉPONSE À L'EXIGENCE DE CONFORMITÉ AVEC LE DROIT INTERNATIONAL DES DROITS DE L'HOMME ?}

Cependant, la responsabilité civile, administrative, voire dans certains cas pénale de l'entreprise en cas de corruption économique, ainsi que les différentes actions qui lui sont demandées et les multiples incitations en faveur de la mise en œuvre des systèmes d'autorégulation susmentionnés, sont cohérentes au regard de ce qui est attendu de l'entreprise par le système universel et régional de protection des droits de l'homme, et par l'Organisation Internationale du Travail (OIT). Certes, ici la motivation ou l'intention ne consiste pas à défendre l'application des principes du libre marché à l'échelle mondiale, mais à garantir la jouissance effective des droits de l'homme et la protection de l'environnement.

La question est généralement traitée sous une rubrique Entreprises et droits de I'homme qui énonce ensuite les « Principes directeurs relatifs aux entreprises et aux droits de l'homme (UNGPs) $»^{6}$. En substance, le document énonce qu'il incombe aux entreprises privées d'empêcher ou de réparer tout dommage pouvant survenir en raison d'atteinte aux droits de l'homme internationalement reconnus, à savoir au minimum ceux figurant dans la Charte internationale des droits de l'homme. Il indique également qu'au regard de la nature de ces obligations, la diligence raisonnable est l'outil le plus adapté. Celui-ci désigne l'ensemble des processus mis en place par les organisations pour identifier leurs

5 En Argentine, cette question figure parmi les préoccupations et les activités des organismes publics. Ainsi, le Bureau anticorruption a publié en 2018 les « Lignes directrices sur l'intégrité pour les entreprises et autres entités juridiques » qui, depuis 2019, disposent d'un "Guide complémentaire » à destination des petites et moyennes entreprises.

6 Adopté dans le cadre du Conseil des droits de l'homme des Nations Unies par la Résolution 17/4 du 16 juin 2011. Le document comprend 31 principes classés en trois groupes : a) Obligations de protéger les droits de l'homme incombant à l'État; b) Responsabilité incombant aux entreprises de protéger les droits de l'homme; et c) La nécessité de faciliter l'accès à des voies de recours pour les victimes de violations causées par des entreprises. Pour une étude approfondie et détaillée, voir "La responsabilité des entreprises de respecter les droits de l'homme: guide interprétatif », du Haut-Commissariat des Nations Unies aux Droits de l'Homme (HCDH).

7 Cela comprend les droits énoncés dans la Déclaration universelle des droits de l'homme, le Pacte international relatif aux droits civils et politiques (PIDCP) et le Pacte international relatif aux droits économiques, sociaux et culturels (PIDESC). 
incidences sur ces droits, prévenir ces incidences, en atténuer les effets et rendre compte de la manière dont elles y remédient. Ces incidences négatives potentielles ou réelles, peuvent être causées en tout ou en partie par leurs activités, ou par les activités liées à leurs opérations, à leurs produits ou aux services fournis dans le cadre de leurs relations commerciales ${ }^{8}$.

La précieuse contribution du document relatif aux «Principes directeurs » s'inscrit dans la tendance au renforcement et à la hiérarchisation des droits économiques, sociaux et culturels. Ce phénomène repose sur l'idée d'universalité, d'indivisibilité, d'interdépendance et d'interrelation de tous les droits de l'homme et des libertés fondamentales'. Comme l'indiquent les «Principes directeurs», la violation - quelle qu'elle soit - de l'un des droits de I'homme engage automatiquement la responsabilité de l'entreprise privée directement ou indirectement concernée.

Les "Principes directeurs » relatifs aux entreprises et aux droits de l'homme ne sont pas en eux-mêmes contraignants et n'engagent en aucune façon la responsabilité internationale des États. Toutefois, ils stipulent clairement que les Gouvernements et les entreprises sont tenus de respecter - et de faire respecter - les droits de l'homme. Ces derniers étant des principes fondamentaux, adoptés dans le cadre des Nations Unies et défendus par le Conseil des droits de l'homme, il ne serait pas raisonnable de nier ou de contester leur portée.

Par ailleurs, il convient de souligner que les «Principes directeurs», constituent une véritable référence mondiale en la matière, y compris pour les instruments les plus importants du Système international de protection des droits de l'homme. Ainsi, le Comité des Droits Economiques, Sociaux et Culturels en a tenu compte dans plusieurs de ses observations générales ${ }^{10}$, tout comme le Comité des droits de l'enfant ${ }^{11}$. À leur tour, la Déclaration de principes tripartite de $\mathrm{I}^{\prime} \mathrm{OIT}^{12}$ sur les entreprises multinationales et la politique sociale, et les Principes directeurs de l'OCDE à l'intention des entreprises multinationales ${ }^{13}$, ont été mis à jour suite à l'adoption de ces « Principes directeurs». D'autre part, en vertu d'un mandat exprès du Conseil des droits de l'homme, il incombe au Haut-Commissaire des Nations Unies aux Droits de l'Homme (HCDH) et au Groupe de travail Entreprises et droits de l'homme ${ }^{14}$

8 Rapport du Groupe de travail sur la question des droits de l'homme et des sociétés internationales et autres entreprises, en date du 16/07/2018. Comme le précise le document des Nations Unies sur les Principes directeurs relatifs aux entreprises et aux droits de l'homme, les « relations commerciales » s'entendent par des « relations de l'entreprise avec ses partenaires commerciaux, les entités de sa chaîne de valeur, et toute autre entité non étatique ou étatique directement liée à ses activités, ses produits ou ses services commerciaux " (commentaire sur le principe $n^{\circ} 13$ ).

9 Voir le Préambule du Protocole Facultatif (PF-PIDESC) du 10/12/2008.

10 Le document est mentionné dans l'Observation générale ${ }^{\circ} 23$ (2016) sur le droit à des conditions de travail justes et favorables (article 7 du Pacte international relatif aux droits économiques, sociaux et culturels), et également dans l'Observation générale $n^{\circ} 24$ (2017) sur les obligations des États au titre du Pacte dans le cadre des activités commerciales.

11 Observation générale n 16 (2013), sur les obligations de l'État concernant l'impact du secteur des entreprises sur les droits de l'enfant.

12 Adoptée initialement en 1977 et révisée pour la dernière fois en 2017.

13 Mis à jour en 2011, il comporte aujourd'hui un chapitre fondé sur les « Principes directeurs » et intègre le concept de diligence raisonnable.

14 Groupe créé par le Conseil des Droits de l'Homme en 2011 (Résolution n 17/4) et composé de cinq experts indépendants. 
de promouvoir leurs recommandations ${ }^{15}$. De même, au sein du Système Interaméricain des Droits de l'Homme (SIDH), la Commission des droits de l'homme a fait allusion aux Principes directeurs dans son rapport du 31 décembre 2015 intitulé «Peuples autochtones, communautés d'ascendance africaine et ressources naturelles : la protection des droits de I'homme dans le contexte de l'extraction, de l'exploitation et du développement »; la CIDH les ayant cités dans plusieurs de ses prises de position ${ }^{16}$.

Enfin, il est à signaler que la Colombie, le Chili, l'Argentine et le Pérou ont repris les exigences des «Principes directeurs » dans leur Plan d'action national pour la promotion et la protection des droits de l'homme ${ }^{17}$. Cette initiative répond à une demande formulée par le Comité des droits de l'homme, visant à ce que chaque État intègre dans son Plan d'action national ou tout autre dispositif similaire, des éléments facilitant la mise en œuvre des Principes directeurs susmentionnés ${ }^{18}$.

\section{II - L'IMPACT DU DROIT INTERNATIONAL DES DROITS DE L'HOMME SUR LES SYSTĖMES JURIDIQUES NATIONAUX}

\section{A - Les ATTENTES VIS-À-VIS dES ETATS d'AMÉRIQUe LATINE}

De manière générale, les États d'Amérique latine se sont engagés à appliquer le droit international des droits de l'homme. Celui-ci consiste en la somme des principaux instruments normatifs du système universel et régional des droits de l'homme, auxquels s'ajoute l'activité interprétative des organes de contrôle correspondants ${ }^{19}$. Cet engagement se traduit, au sein des États et de leurs systèmes normatifs particuliers, par l'adoption et l'adhésion pleine et entière à la doctrine du bloc de constitutionnalité dont la finalité conduit à doter les instruments issus du droit international des droits de l'homme d'un statut normatif supérieur (constitutionnel) à toute autre norme de l'ordre interne.

Naturellement, le respect des droits de l'homme va au-delà de la décision de l'État d'adhérer et de participer aux instances internationales, et dépasse également la décision politique d'approuver et de ratifier des textes normatifs pour leur attribuer ensuite une position privilégiée dans la pyramide normative. En effet, le respect des droits de l'homme exige une action beaucoup plus large et plus profonde, qui nécessite un processus global

15 Contribution de l'ensemble du système des Nations Unies à la promotion du Programme relatif aux entreprises et aux droits de l'homme, ainsi qu'à la diffusion et à l'application des Principes directeurs relatifs aux entreprises et aux droits de l'homme (A/HRC/RES/21/5).

16 Affaire Ximenes Lopes. c. Brésil du 07/04/2006 ; affaire Albán Cornejo et autres. c. Équateur du 22/11/2007 ; affaire Suárez Peralta c. Équateur du 21/05/2013; affaire Kaliña et Lokono Villages c. Surinam du 25/11/2015; et affaire Travailleurs de l'Hacienda Brasil Verde c. Brésil du 20/10/2016.

17 La conception et la mise en œuvre de ces plans est une recommandation issue de la Conférence mondiale sur les droits de l'homme du 25 juin 1993 et est expressément mentionnée au § 71 de la Déclaration et du Programme d'action de Vienne qui y a été adopté.

18 Résolution A/HRC/26/L. 1 du 23/06/2014.

19 En ce qui concerne les droits économiques, sociaux et culturels (DESC), il convient de souligner la ratification du PIDESC par l'Argentine, la Bolivie, le Brésil, le Chili, la Colombie, le Costa Rica, l'Équateur, le Salvador, le Guatemala, le Honduras, le Mexique, le Nicaragua, le Panama, le Paraguay, le Pérou et l'Uruguay, et de son Protocole facultatif par l'Argentine, la Bolivie, le Costa Rica, I'Équateur, le Salvador, le Honduras, I'Uruguay et le Venezuela. 
de transformation, de reconfiguration et de sensibilisation ; un processus transcendant à bien des égards et axé sur la conduite des fonctions administratives, législatives et juridictionnelles. Ainsi, il s'agit d'un renouvellement pertinent consacré ou destiné à la poursuite des objectifs inhérent aux principes du droit international des droits de l'homme dans la sphère interne. Dès lors, plus qu'une série d'actions spécifiques et déterminées, il s'agit d'un engagement dans un processus de modification et d'adaptation.

D'un point de vue transnational et plus particulièrement s'agissant de l'Amérique latine, le $\mathrm{SIDH}^{20}$ a joué un rôle moteur important dans ce processus, à travers notamment la Convention américaine relative aux droits de l'homme (CADH), son instrument normatif le plus important. Ratifiée par tous les États d'Amérique latine, à l'exception de Cuba, cette Convention est assortie d'un protocole additionnel relatif aux droits économiques, sociaux et culturels (Protocole de San Salvador) ${ }^{21}$. Non seulement la CADH énonce les droits de l'homme inviolables et contraignants pour les États parties (y compris les États qui se sont engagés à respecter les droits humains et les libertés fondamentales en vertu de la Charte de l'OEA ${ }^{22}$ et de la Charte démocratique interaméricaine ${ }^{23}$ ), mais elle impose également aux États de mettre leur système législatif et leurs pratiques en conformité avec la Convention ${ }^{24}$. En outre, la CADH met en place les organes chargés de veiller à ce que ses exigences soient respectées : la Commission IDH et la Cour IDH ${ }^{25}$, dont la tâche est subsidiaire - ou complémentaire - au devoir initial de conformité qui pèse sur chaque État partie à la $\mathrm{CADH}^{26}$.

Ce processus est unique et vise exclusivement à déterminer si un État partie a violé ou non les droits énoncés par la Convention et, dans l'affirmative, s'il doit réparer les dommages causés par son comportement illicite et dans quelle mesure. L'organe transnational ne cherche donc pas à résoudre un conflit entre les parties, il veille plutôt au respect du droit conventionnel par des décisions contraignantes ${ }^{27}$.

Le processus contentieux qui a lieu dans le cadre transnational du SIDH a certainement été novateur à bien des égards et avec une approche que l'on pourrait qualifier parfois d'intransigeante. C'est d'ailleurs pour cette raison que des États ont à certaines périodes pris leurs distances ${ }^{28}$. La gravité des questions en jeu explique sans aucun doute ce type

20 Le Système Interaméricain des Droits de l'Homme (SIDH) a été institué par l'Organisation des États Américains (OEA). II repose sur la Déclaration américaine des droits et devoirs de l'homme de 1948, et sur le texte de la Convention américaine relative aux droits de l'homme (CADH) de 1969.

21 Ratifié par l'Argentine, la Bolivie, le Brésil, la Colombie, le Costa Rica, l'Équateur, le Salvador, le Guatemala, le Mexique, le Panama, le Paraguay, le Pérou, l'Uruguay et le Venezuela.

22 Art. 3.1.

23 Art. 3, 7, 8 et 9 .

24 Art. 2 de la CADH.

25 Bien que la grande majorité des États d'Amérique latine aient ratifié la CADH, ceux qui l'ont fait n'ont pas tous reconnu la compétence de la Cour IDH (par exemple la République dominicaine) ou ont émis certaines réserves quant aux actions de la Commission IDH (par exemple la Bolivie, le Brésil, le Mexique et le Paraguay).

26 Commission IDH, Affaire 11.671 Carlos García Saccone c. Argentine, Rapport 11/98.

27 Voir la Cour IDH, Responsabilité internationale pour la promulgation et l'application de lois en violation de la Convention (art. 1 et 2 de la Convention américaine relative aux droits de l'homme), OC-14/94 du 9 décembre 1994, Série A, nº 14, §35.

28 O. A. Gozaini, Tratado de Derecho Procesal Civil, Editorial La Ley, Buenos Aires, 2019, p. 64. 
d'intervention avec un tel élan transformateur, lequel aurait dû servir de catalyseur pour étendre, élargir, relativiser, voire reconfigurer et adapter les règles habituellement acceptées au sein des processus juridictionnels, ou encore pour formuler de nouveaux principes plus conformes à la nature et aux objectifs de ces processus. A titre d'exemples, citons notamment la recherche d'une vérité réelle et pas seulement formelle ou procédurale ; la déclaration de non-applicabilité de la prescription en matière de crimes contre l'humanité ; la consécration du devoir impératif d'investigation des États ; la non-application de la présomption d'innocence dans certains cas; l'assouplissement de la charge de la preuve en général - compensé ici et là par le devoir de coopération - ou les enquêtes menées par les agences du SIDH.

\section{B - L'IMPACT SUR L'ACTIVITÉ JURIDICTIONNELLE}

Le droit international des droits de l'homme se heurte et interfère fortement avec l'activité juridictionnelle au sein des États et ce, pour deux raisons principales. D'une part, si I'on observe la sphère transnationale, et en particulier l'activité des organisations du SIDH, tant sur le fond (en termes de contenu et d'élargissement des droits) que sur la forme (à savoir les innovations ou ajustements énoncés plus haut), le droit international apparaît comme une source d'inspiration potentielle pour l'exercice du pouvoir juridictionnel au sein des différents États. D'autre part, l'universalisation, la complexité, le développement et la sophistication des droits de l'homme, permettent d'accéder à une réelle compréhension d'un point de vue juridique de toute relation intersubjective, ce qui est particulièrement évident dans les relations asymétriques, comme celles du travail dépendant ${ }^{29}$. En ce sens, toute conduite ou tout comportement intersubjectif risque toujours et dans une certaine mesure, d'avoir une incidence, positive ou négative, sur un droit fondamental quelconque. II faut donc une analyse qui tienne compte de cette réalité, et un contrôle de conventionnalité exercé par une autorité judiciaire (contrôle diffus) voire même d'office ${ }^{30}$. II semble que ces deux aspects combinés expliquent et décrivent avec suffisamment d'éloquence le fonctionnement des juridictions selon l'influence exercée par le SIDH, notamment dans les situations qui n'impliquent pas de violations graves ou systématiques des droits de I'homme.

Le droitinternational des droits de l'homme a ainsi transforméla pratique juridictionnelle, la compréhension du phénomène juridique (en général) et renforcé, au sein de chaque État, la pertinence des principes d'un État constitutionnel et conventionnel fondé sur la primauté du droit. Cette situation se traduit par la constitutionnalisation et la conventionnalisation virtuelles du droit privé, la perception de la législation interne comme simple incarnation du droit constitutionnel et conventionnel, et l'assurance que le juge disposera de plus d'outils pour réaliser son propre idéal de justice. C'est ici que l'utilisation de critères évolutifs dans

29 L'obligation de respecter les droits de l'homme comprend également le respect de la vie privée, ce que l'on appelle l'« efficacité horizontale ». Voir CIDH, Affaire Lagos del Campo c. Pérou, 31/08/2017; CIDH, Avis consultatif 18/03, Condition juridique et droits des migrants sans papiers, $17 / 09 / 2013$.

30 Le contrôle de conventionnalité est développé dans la jurisprudence de la $\mathrm{CIDH}$, voir Almonacid Arellano c. Chili du 26/09/2006 ; Travailleurs licenciés du Congrès (Aguado Alvaro et al.) c. Pérou du 24/11/2006 ; Boyce et autres c. Barbade du 20/11/2007; Radilla Pacheco c. Mexique du 23/09/2009; et dans son Avis consultatif OC-21/14/ du 19/08/2014 sur les droits et garanties des enfants dans le contexte de la migration et/ou ayant besoin d'une protection internationale. 
l'attribution de la charge de la preuve est mise en avant et pertinente, avec l'application du principe de coopération, de solidarité et de diligence des parties concernées ${ }^{31}$; et que la recherche de la preuve par les juges eux-mêmes est reconnue et légitimée ${ }^{32}$.

En ce sens, il faut avoir conscience de l'importance cruciale de la prévention en matière de protection des droits dits homogènes ou d'incidence collective. En effet, l'obligation légale de prévention est d'autant plus justifiée là où l'incitation à la protection des droits est la plus faible ${ }^{33}$. Le domaine environnemental est particulièrement concerné, compte tenu de la façon dont les dommages sont causés et de ceux qui y contribuent. La volonté de lutter efficacement contre les dommages irréparables dans le domaine des droits de I'homme et de l'environnement donne lieu au développement du recours au principe de précaution, ou encore à la mise en œuvre de processus collectifs ${ }^{34}$; de même en droit privé général ou commun, et en particulier en droit de la responsabilité35, la tendance est de ne pas exiger la prise en compte du facteur subjectif (faute ou malveillance) pour engager la responsabilité ${ }^{36}$, mais de privilégier le droit de la victime à obtenir réparation, ou d'établir de nouveaux cas de responsabilité objective ${ }^{37}$, etc.

\section{Conclusion}

Toutes ces nouvelles manifestations et expressions contribuent à un soi-disant néo-procéduralisme ${ }^{38}$ qui exalte la figure du Juge, favorise et stimule un volontarisme judiciaire croissant et un activisme orienté vers la mise en œuvre des droits de l'homme, la protection et la préservation de l'environnement.

31 Voir l'arrêt de la Cour suprême de justice de la Nation du Mexique (Amparo de Revisión $n^{\circ}$ 307/2006), en matière d'environnement, où le renversement de la charge de la preuve a été établi sur la base du principe de précaution. En Argentine, voir la jurisprudence de la Cour suprême de justice concernant la charge spéciale de la preuve applicable en particulier aux cas de discrimination, affaire Pellicori, Liliana Silvia c. Colegio Público de Abogados de la Capital Federal du 15/11/2011.

32 Voir en Uruguay l'article 139.2 du Code général de procédure ; en Argentine, l'article 1735 du Code civil et commercial ; au Brésil, l'article 373 du Code de procédure civile ; en Colombie, l'article 167 du Code général de procédure.

33 R. L. Lorenzetti, Código Civil y Comercial de la Nación comentado, Ed. Rubinzal-Culzoni, $2014-$ 2019, vol. VIII, p. 283.

34 En Argentine, il s'agit d'une jurisprudence de la Cour suprême (Halabi, Ernesto s/amparo du 24/2/2009) avec application aux cas de discrimination à l'embauche (Sisnero, Mirtha Graciela et autres c/Taldelva SRL et autres s/amparo du 20/05/2014).

35 En Argentine, les articles 1710 à 1713 du Code civil et commercial unifié énoncent l'obligation pour toute personne d'éviter tout préjudice injustifié, dans la mesure où il dépend d'elle, et réglementent l'« action préventive », qui intervient lorsqu'une action ou une omission anti-juridique rend prévisible la survenance d'un dommage, sa persistance ou son aggravation. Au Chili, l'article 2333 du Code civil prévoit une action pour dommages contingents, de même qu'en Colombie, au Salvador et en Équateur.

36 En Argentine, le préjudice injustifié permet de présumer l'illégalité du comportement qui en est à l'origine (voir art. 1717 CCyC).

37 Au Chili, l'article 52 de la loi sur l'environnement n 19300 de 1994 établit la présomption de culpabilité.

38 O. A. Gozaini, Conflictos constitucionales y procesales del sistema interamericano de derechos humanos, Ed. Ediar, Buenos Aires, 2009, T. I, p. 32. 


\section{L'AUTORÉGULATION DES ENTREPRISES EN ARGENTINE}

L'activité juridictionnelle, influencée par les principes de l'État de droit constitutionnel et conventionnel, permet d'espérer que la responsabilité directe et non transférable qui pèse sur les entreprises ait comme corolaire une exigence forte et cohérente de diligence raisonnable au sein des organisations. Ceci est particulièrement intéressant car, comme évoqué ci-avant, les systèmes d'autorégulation des entreprises - qui constituent certainement la réponse appropriée aux nombreuses demandes que la communauté mondiale adresse aujourd'hui aux organisations - sont des instruments de conception et de mise en œuvre volontaires, en ce sens qu'il n'existe pas de disposition contraignante les imposant. Le caractère exécutoire tendrait donc à combler ce manque et serait par ailleurs très bénéfique dans la mesure où ces instruments uniques et formidables ne sauraient dépendre de la seule initiative entrepreneuriale, et nécessitent dès lors une action conjointe globale. Cette action devrait être engagée car chaque État assume la responsabilité d'exercer fermement et justement le pouvoir de coercition dont il est doté. En Amérique latine, les conditions sont réunies pour que cela soit possible ${ }^{39}$.

39 À titre d'exemple, voir la décision de la Deuxième Chambre de droit constitutionnel et social de la Cour suprême de justice de la République du Pérou, Casación Laboral n² 25875-2018 TACNA, indemnisation pour dommages et intérêts et autres, du 24/10/2019.

\section{DIEGO MARCELO LEDESMA ITURBIDE}

Avocat spécialiste en droit du travail, Professeur à la Faculté de droit et des sciences économiques de l'Université de Buenos Aires, Co-directeur du projet PDE-9-2019 « Corruption, fraude commerciale et systèmes de conformité dans les organisations " de l'Unité Académique à l'Université de Buenos Aires.

Thèmes de recherche : Systèmes d'autorégulation des entreprises, diligence raisonnable, droit international des droits de l'homme.

\section{Publications:}

D. M. Ledesma Iturbide, " Las razones y el contexto del conflicto sobre condiciones de trabajo ", Revista Trabajo y Seguridad Social, Ed. El Derecho, n³1, Mars 2018.

$\sim$ D. M. Ledesma lturbide et P. R. Arce, « Derecho a la estabilidad y principio de causalidad en el despido en el contexto de un Estado Constitucional de Derecho y Antropocéntrico ", in Contratación laboral y despido - II, Revista de Derecho Laboral, Ed. Rubinzal-Culzoni, 2018/2. 